\title{
Tables of Fibonacci and Lucas Factorizations
}

\author{
By John Brillhart, Peter L. Montgomery, and Robert D. Silverman \\ Dedicated to Dov Jarden
}

\begin{abstract}
We list the known prime factors of the Fibonacci numbers $F_{n}$ for $n \leq 999$ and Lucas numbers $L_{n}$ for $n \leq 500$. We discuss the various methods used to obtain these factorizations, and primality tests, and give some history of the subject.
\end{abstract}

1. Introduction. In the Supplements section at the end of this issue we give in two tables the known prime factors of the Fibonacci numbers $F_{n}, 3 \leq n \leq 999, n$ odd, and the Lucas numbers $L_{n}, 2 \leq n \leq 500$. The sequences $F_{n}$ and $L_{n}$ are defined recursively by the formulas

$$
\begin{array}{lll}
F_{n+2}=F_{n+1}+F_{n}, & F_{0}=0, & F_{1}=1, \\
L_{n+2}=L_{n+1}+L_{n}, & L_{0}=2, & L_{1}=1 .
\end{array}
$$

The use of a different subscripting destroys the divisibility properties of these numbers.

We also have the formulas

$$
F_{n}=\frac{\alpha^{n}-\beta^{n}}{\alpha-\beta}, \quad L_{n}=\alpha^{n}+\beta^{n},
$$

where $\alpha=(1+\sqrt{5}) / 2$ and $\beta=(1-\sqrt{5}) / 2$. This paper is concerned with the multiplicative structure of $F_{n}$ and $L_{n}$. It includes both theoretical and numerical results.

2. Multiplicative Structure of $F_{n}$ and $L_{n}$. The identity

$$
F_{2 n}=F_{n} L_{n}
$$

follows directly from (1.2). Although the Fibonacci and Lucas numbers are defined additively, this is one of many multiplicative identities relating these sequences. The identities in this paper are derived from the familiar polynomial factorization

$$
x^{n}-y^{n}=\prod_{d \mid n} \Phi_{d}(x, y), \quad n \geq 1,
$$

where $\Phi_{d}(x, y)$ is the $d$ th cyclotomic polynomial in homogeneous form.

Define the primitive part $F_{d}^{*}$ of $F_{d}$ to be

$$
F_{d}^{*}= \begin{cases}1, & d=1, \\ \Phi_{d}(\alpha, \beta), & d \geq 2 .\end{cases}
$$

Received February 9, 1987; revised April 27, 1987.

1980 Mathematics Subject Classification (1985 Revision). Primary 11A51, 11B39; Secondary 68-04.

Key words and phrases. Factor tables, Fibonacci, Lucas, factorizations. 
Then we have the factorization

$$
F_{n}=\prod_{d \mid n} F_{d}^{*}, \quad n \geq 1
$$

Here the $F_{d}^{*}$ are rational integers, computable by the inverse formula

$$
F_{d}^{*}=\prod_{\delta \mid d} F_{\delta}^{\mu(d / \delta)}, \quad d \geq 1
$$

where $\mu$ is the Möbius function. The ratio $F_{n}^{\prime}=F_{n} / F_{n}^{*}$ is called the algebraic part of $F_{n}$.

Formula (2.4) reduces factoring $F_{n}$ to factoring the $F_{d}^{*}$ 's. Formula (2.5) shows that the primitive part can be obtained without factoring.

A prime factor of $F_{n}$ (resp. $L_{n}$ ) is called primitive if it does not divide $F_{k}$ (resp. $L_{k}$ ) for $1 \leq k<n$; otherwise it is called algebraic. A composite factor of $F_{n}$ is also called algebraic if it is a product of prime algebraic factors. Any prime divisor of $F_{n}^{\prime}$ (resp. $L_{n}^{\prime}$ ) is necessarily algebraic, but under certain circumstances a prime divisor of $F_{n}^{*}$ (resp. $L_{n}^{*}$ ) is not primitive. Such an algebraic prime factor $p$ of $F_{n}^{*}$ (resp. $L_{n}^{*}$ ) is called intrinsic and is listed as $p^{*}$ in these tables. This occurs exactly when $n=p^{r} m, r \geq 1$, where $p$ is a primitive factor of $F_{m}$ (resp. $L_{m}$ ). In this case $p$ always divides $F_{n}^{*}$ (resp. $L_{n}^{*}$ ) to just the first power.

Example. The factorization of $F_{105}$, given by (2.4), is

$$
F_{105}=\prod_{d \mid 105} F_{d}^{*}=F_{1}^{*} F_{3}^{*} F_{5}^{*} F_{7}^{*} F_{15}^{*} F_{21}^{*} F_{35}^{*} F_{105}^{*}
$$

This factorization is abbreviated in Table 2 as

$$
105(3,5,7,15,21,35) 8288823481 .
$$

Here the numbers within the parentheses are the subscripts of the algebraic factors $F_{d}^{*}, 1<d<105$. (The factor $F_{1}^{*}=1$ is omitted.) The primitive part $F_{105}^{*}=$ 8288823481 is given after the parentheses. The lines in Table 2 corresponding to the numbers inside the parentheses are:

$$
\begin{aligned}
3 & \underline{2} \\
5 & \underline{5} \\
7 & \underline{13} \\
15 & (3,5) \underline{61} \\
21 & (3,7) \underline{421} \\
35 & (5,7) \underline{141961}
\end{aligned}
$$

The factorization of $F_{105}$ is then obtained by collecting the primitive prime factors from their respective lines. These follow the parentheses (if any) on the seven lines and are underlined above for emphasis. Thus,

$$
F_{105}=2 \cdot 5 \cdot 13 \cdot 61 \cdot 421 \cdot 141961 \cdot 8288823481 \text {. }
$$

Because of (2.1), the algebraic multiplicative structure for $L_{n}$ can be derived directly from that of $F_{2 n}$. Let $n=2^{s} m$, where $m$ is odd. Then

$$
L_{n}=\prod_{d \mid m} L_{2, d}^{*}, \quad n \geq 1,
$$


where

$$
L_{2^{s} d}^{*}=F_{2^{s+1} d}^{*}=\prod_{\delta \mid d} L_{2^{s} \delta}^{\mu(d / \delta)}, \quad d \geq 1 .
$$

The primitive part of $L_{n}$ is $L_{n}^{*}=F_{2 n}^{*}$. The algebraic part of $L_{n}$ is

$$
L_{n}^{\prime}=L_{n} / L_{n}^{*}
$$

Furthermore, as a result of a generalization by Lucas of a special identity discovered by Aurifeuille, we also have for odd $n$

$$
\begin{aligned}
\frac{L_{5 n}}{L_{n}} & =\frac{\alpha^{5 n}+\beta^{5 n}}{\alpha^{n}+\beta^{n}}=\alpha^{4 n}-\alpha^{3 n} \beta^{n}+\alpha^{2 n} \beta^{2 n}-\alpha^{n} \beta^{3 n}+\beta^{4 n} \\
& =\left(\alpha^{2 n}-3 \alpha^{n} \beta^{n}+\beta^{2 n}\right)^{2}+5 \alpha^{n} \beta^{n}\left(\alpha^{n}-\beta^{n}\right)^{2} \\
& =\left(5 F_{n}^{2}+1\right)^{2}-25 F_{n}^{2} \\
& \left.=\left(5 F_{n}^{2}+5 F_{n}+1\right)\left(5 F_{n}^{2}-5 F_{n}+1\right) \text { (using } \alpha \beta=-1 \text { and } \alpha-\beta=\sqrt{5}\right) .
\end{aligned}
$$

Consequently, we have the special Aurifeuillian factorization

$$
L_{5 n}=L_{n} A_{5 n} B_{5 n}, \quad n \text { odd }
$$

where

$$
A_{5 n}=5 F_{n}^{2}-5 F_{n}+1, \quad B_{5 n}=5 F_{n}^{2}+5 F_{n}+1 .
$$

This decomposition means that these $L_{5 n}$ 's have two different algebraic factorizations. For example, from (2.6) and (2.9)

$$
L_{105}=\prod_{d \mid 105} L_{d}^{*}=L_{1}^{*} L_{3}^{*} L_{5}^{*} L_{7}^{*} L_{15}^{*} L_{21}^{*} L_{35}^{*} L_{105}^{*}
$$

and

$$
L_{105}=L_{21} A_{105} B_{105} .
$$

Primitive parts $A_{n}^{*}$ and $B_{n}^{*}$ can also be defined for $A_{n}$ and $B_{n}$. Let $n \geq 1$ be odd and set $n=5^{s} m, s \geq 0,5+m$. Let $\varepsilon_{d}=\frac{1}{2}\left(1+\left(\frac{d}{5}\right)\right)$, where $\left(\frac{d}{5}\right)$ is the Legendre symbol. Let

$$
\begin{aligned}
& A_{5 n}^{*}=\prod_{d \mid m}\left[\left(A_{5 n / d}\right)^{\varepsilon_{d}}\left(B_{5 n / d}\right)^{1-\varepsilon_{d}}\right]^{\mu(d)} \\
& B_{5 n}^{*}=\prod_{d \mid m}\left[\left(A_{5 n / d}\right)^{1-\varepsilon_{d}}\left(B_{5 n / d}\right)^{\varepsilon_{d}}\right]^{\mu(d)}
\end{aligned}
$$

(Here $A_{5 n}^{*}$ and $B_{5 n}^{*}$ are rational integers such that $\left(A_{5 n}^{*}, B_{5 n}^{*}\right)=1$ and $L_{5 n}^{*}=$ $A_{5 n}^{*} B_{5 n}^{*}$.) Then

$$
\begin{aligned}
& A_{5 n}=\prod_{d \mid m}\left(A_{5 n / d}^{*}\right)^{\varepsilon_{d}}\left(B_{5 n / d}^{*}\right)^{1-\varepsilon_{d}}, \\
& B_{5 n}=\prod_{d \mid m}\left(A_{5 n / d}^{*}\right)^{1-\varepsilon_{d}}\left(B_{5 n / d}^{*}\right)^{\varepsilon_{d}} .
\end{aligned}
$$

Thus, in the above example we have

$$
A_{105}=A_{5}^{*} B_{15}^{*} B_{35}^{*} A_{105}^{*}, \quad B_{105}=B_{5}^{*} A_{15}^{*} A_{35}^{*} B_{105}^{*} .
$$


Since $A_{5}^{*}=A_{15}^{*}=1$, these are omitted in Table 3 , while $B_{5}^{*}$ is written as $L_{5}^{*}$ and $B_{15}^{*}$ as $L_{15}^{*}$.

Those Lucas numbers which do not have an Aurifeuillian factorization appear in the tables in the same format as the Fibonacci factorizations. However, the Aurifeuillian factorizations appear in an expanded format. For example, the above factorization appears as:

$$
\begin{aligned}
& 105(3,7,21) A \cdot B \\
& A(15,35 B) 21211 \\
& B(5,35 A) 767131 .
\end{aligned}
$$

The list of numbers immediately after the index 105 indicate that $L_{105}$ has the algebraic factors $L_{3}^{*}, L_{7}^{*}$, and $L_{21}^{*}$. Furthermore, $A_{105}^{*}$ has algebraic factors $L_{15}^{*}$ and $B_{35}^{*}$, while $B_{105}^{*}$ has algebraic factors $L_{5}^{*}$ and $A_{35}^{*}$. In computing $A_{n}^{*}$ and $B_{n}^{*}$, the following result is sometimes useful $[9$, p. 16]:

TheOREM 1 (CROSSOVER ThEOREM). For odd $k, n \geq 1$ where $(5, k)=1$ and $\left(\frac{k}{5}\right)$ is the Jacobi symbol,

$$
\begin{aligned}
& \text { if }\left(\frac{k}{5}\right)=1, \text { then } A_{5 n} \mid A_{5 k n} \text { and } B_{5 n} \mid B_{5 k n} \\
& \text { if }\left(\frac{k}{5}\right)=-1, \text { then } A_{5 n} \mid B_{5 k n} \text { and } B_{5 n} \mid A_{5 k n} .
\end{aligned}
$$

The tables are organized using formulas (2.4) and (2.6). As a result, no prime factor appears explicitly more than once in the tables (except intrinsic factors and the repeated factor 2 of $L_{3}$ ). Where space permits, we list the known factors in their entirety on a single line. We list all prime factors of 25 digits or less, carrying over to a second line, without breaking the factor, when necessary. All other factors are listed as either Pxx or Cxx, indicating respectively a prime or a composite cofactor of $x x$ digits. When a factorization is incomplete, we leave space on the line for new factors to be inserted by hand.

3. Factorization Methods. A variety of methods have been used to effect the factorizations given herein. These include the Pollard $p-1$ and Brent-Pollard Rho methods [13], the analogous $p+1$ method [19], the Continued Fraction (CFRAC) method of Morrison and Brillhart [14], Pomerance's Quadratic Sieve (QS) method [8], along with its extensions and improvements (MP-QS) [17], [18], and Lenstra's Elliptic Curve Method (ECM) [11], [13]. Of course, many of the smaller prime factors are quite old, and were originally found by trial division or the difference of squares method.

Some of the methods utilize the form of the prime divisors given by the following theorems [9, p. 11].

THEOREM 2. Let $n$ be odd and let $p$ be an odd, primitive prime divisor of $F_{n}$. Then

(i) $p \equiv 1 \bmod 4$.

(ii) if $p \equiv \pm 1 \bmod 10$, then $p \equiv 1 \bmod 4 n$.

(iii) if $p \equiv \pm 3 \bmod 10$, then $p \equiv 2 n-1 \bmod 4 n$. 
THEOREM 3. Let $n$ be positive and let $p$ be an odd, primitive prime divisor of $L_{n}$. Then

(i) if $p \equiv \pm 1 \bmod 10$, then $p \equiv 1 \bmod 2 n$.

(ii) if $p \equiv \pm 3 \bmod 10$, then $p \equiv-1 \bmod 2 n$.

4. Primality Testing. In [9, p. 36], Brillhart gave the following results of primality tests on the Fibonacci and Lucas numbers: $F_{n}, 3 \leq n<1000$, is prime if and only if $n=3,4,5,7,11,13,17,23,29,43,47,83,131,137,359,431,433,449,509,569$, $571 ; L_{n}, 0 \leq n \leq 500$, is prime if and only if $n=0,2,4,5,7,8,11,13,16,17,19,31$, $37,41,47,53,61,71,79,113,313,353$. More recently, H. C. Williams has discovered that $F_{2971}, L_{503}, L_{613}, L_{617}$ and $L_{863}$ are also prime. Williams also states that $F_{4723}$ and $F_{5387}$ are probable primes [21].

For $F_{n}$ to be prime, $n \geq 5$, it is necessary, but not sufficient, that $n$ be prime. Similarly, $L_{n}$ can be prime only when $n$ is prime or a power of 2 . There are several identities that can be used for primality proofs if one should find either $F_{n}$ or $L_{n}$ or their primitive parts to be probable primes. These identities are useful because in proving $N$ prime, the methods of [5] depend upon auxiliary factorizations of $N \pm 1$. For the Fibonacci numbers we have $[9$, p. 95]:

$$
F_{4 k+1}-1=F_{k} L_{k} L_{2 k+1}, \quad F_{4 k+3}-1=F_{k+1} L_{k+1} L_{2 k+1}
$$

and

$$
F_{4 k+1}+1=F_{2 k+1} L_{2 k}, \quad F_{4 k+3}+1=F_{2 k+1} L_{2 k+2} .
$$

For the Lucas numbers we have

$$
L_{4 k}-1=L_{6 k} / L_{2 k}, \quad L_{4 k}+1=\left(L_{2 k}-1\right)\left(L_{2 k}+1\right)
$$

and

$$
\begin{array}{ll}
L_{4 k+1}-1=5 F_{k} L_{k} F_{2 k+1}, & L_{4 k+3}-1=L_{2 k+1} L_{2 k+2}, \\
L_{4 k+1}+1=L_{2 k} L_{2 k+1}, & L_{4 k+3}+1=5 L_{k+1} F_{k+1} F_{2 k+1} .
\end{array}
$$

For the Lucas Aurifeuillians we have

$$
\begin{array}{ll}
A_{5 k}-1=5 F_{k}\left(F_{k}-1\right), & B_{5 k}-1=5 F_{k}\left(F_{k}+1\right), \\
A_{5 k}+1=\left(L_{k-1}-1\right)\left(L_{k+1}-1\right), & B_{5 k}+1=\left(L_{k-1}+1\right)\left(L_{k+1}+1\right) .
\end{array}
$$

The use of these formulas is apparent. They break the factorizations of $F_{n} \pm 1$ and $L_{n} \pm 1$ into factorizations of smaller $F_{n}$ 's and $L_{n}$ 's and thus facilitate the primality test. There are a number of additional formulas of a similar kind for $F_{n}^{*} \pm 1$ and $L_{n}^{*} \pm 1$.

All factors and cofactors in Tables 2 and 3 with fewer than 85 digits, and not labelled as Cxx, have been proved prime by Silverman using the methods presented in [5, Section 3] and [20]. These methods depend upon auxiliary factorizations of $p-1, p+1, p^{2}+1, p^{2}+p+1$, and $p^{2}-p+1$. If these cyclotomic polynomials have enough small prime factors, then the methods produce very fast proofs of primality along with a compact certificate which can later be used to verify the proof. Andrew Odlyzko has proved all of the remaining probable prime cofactors to be prime using an implementation of the Cohen-Lenstra algorithm [6]. 
5. History of Tables. Brillhart found many small factors (up to 10 digits) by a direct search program, using Theorems 2 and 3 to restrict the search range for trial division [1], [2]. He later programmed a difference of squares method with modular exclusion to factor $F_{169}, L_{131}, L_{133}, L_{134}, L_{158}, L_{173}$, and $L_{237}$.

In 1968 Brillhart used D. H. Lehmer's delay line sieve DLS 127 at U. C. Berkeley [10] to factor $F_{255}, L_{166}, L_{214}, L_{252}$, and $L_{258}$, again using a difference of squares with modular exclusion. The most remarkable of these factorizations,

$$
F_{255}^{*}=20778644396941 \cdot 20862774425341,
$$

was found in just 40 seconds. Although these two factors are very close, there is no known formula which can account for this factorization.

Between 1970 and 1973, Brillhart and Morrison found a large number of complete factorizations using the continued fraction method, CFRAC, on an IBM 360/91 at UCLA [9], [14].

Starting in 1974, J. L. Selfridge and Marvin C. Wunderlich used an improved version of the UCLA program on an IBM 360/65 at NIU in Dekalb, Illinois to factor many 37-41 digit cofactors. They also implemented the first stage of Pollard's justdiscovered $p-1$ method, and found many new factors. Earl Ecklund and Brillhart programmed and used the first stage of the $p+1$ method as well $[5, \mathrm{p}$. xlii].

H. C. Williams [19] applied the $p \pm 1$ methods to 174 composite Fibonacci and Lucas cofactors which had at most 80 digits.

Thorkil Naur ran the $p-1$ and Pollard Rho methods on $F_{n}$ for odd $n, 1 \leq n \leq$ 399 , and on $L_{n}$ for $0 \leq n \leq 500$. When a factor was at most 53 digits, he completed it via CFRAC. His book [15] and paper [16] list several new factorizations which are included herein.

Montgomery, between 1983 and 1986, applied the methods of [13] to all composite table entries, using idle time on a VAX/780, two VAX/750's and a CDC 7600. He found about 200 previously unknown factors of 11 to 36 digits. Over half of these were found by ECM. He used 10 elliptic curves with limits of $10^{4}$ and $6 \cdot 10^{5}$, another ten curves with limits of $1.6 \cdot 10^{4}$ and $10^{6}$, and a third set of ten curves with limits of $3.2 \cdot 10^{4}$ and $2 \cdot 10^{6}$. Often he used four, five or more sets, but the work is uneven (many more curves were used on the Lucas numbers than on the Fibonacci numbers). Montgomery [13, Section 6] also ran $p+1$ with an initial value (seed) of $15 / 8 \bmod N$ using limits of $3 \cdot 10^{5}$ and $10^{7}$, and again with a seed of $23 / 11 \bmod N$ using limits of $2 \cdot 10^{6}$ and $10^{8}$. If $P \equiv 15 / 8 \bmod N$, then $P^{2}-4 \equiv-31 / 64 \bmod N$ will be a quadratic residue precisely when -31 is a quadratic residue, so this will find a factor of $p$ if $p-\left(\frac{-31}{p}\right)$ is highly composite; this includes cases where 31 divides whichever of $p \pm 1$ is highly composite. The seed of 23/11 $\bmod N$ catches cases where $p-\left(\frac{5}{p}\right)$ is highly composite. By Theorems 2 and 3 , if $p \mid F_{n}^{*}$ ( $n$ odd) or $p \mid L_{n}^{*}$, then $p-\left(\frac{5}{p}\right)$ is divisible by $2 n$, so the latter case occurs frequently. However, these runs did miss some primes $p$ for which $p+1$ is highly composite, such as the factor

$$
2170208701449020077201=2 \cdot 7 \cdot 12583 \cdot 55807 \cdot 424267 \cdot 520309-1
$$

of $F_{795}$ (found by MP-QS; -31 is a nonresidue, but the limits were not high enough on that run). 
Davis and Holdridge [7], in 1984, completed the factorizations of four cofactors $\left(F_{277}, L_{362}, L_{370}\right.$, and $\left.L_{471}\right)$ of 57 to 58 digits, using QS on a CRAY 1 S.

Silverman, between 1983 and 1986, ran $p-1$ with limits of $3 \cdot 10^{6}$ and $5 \cdot 10^{7}$ on the entire Lucas table and on the Fibonacci table to $F_{499}$. He also ran $p-1$ with limits of $2 \cdot 10^{5}$ and $3 \cdot 10^{6}$ on the Fibonacci table from $F_{501}$ to $F_{999}$. This work was accomplished on a Micro-VAX/1 and found about 80 new factors. Some runs with ECM on the Lucas table using the same machine revealed no new factors. Silverman also completed the factorizations of all cofactors below 73 digits, and several larger ones, using either CFRAC or MP-QS [17], [18] on a combination of VAX/780's and SUN-3/75's. The larger factorizations were accomplished using a parallel implementation of MP-QS on a network of SUN's.

6. Accuracy and Completeness of Tables. Montgomery and Silverman independently verified each entry in the main tables. They checked that

- Each listed factor divides the number and is a prime or probable prime.

- The proper list of algebraic (including intrinsic) factors appears

- The primitive prime factors appear in ascending order.

- If no cofactor is given, the list of factors is complete.

- If a cofactor is labelled as Cxx, then it is indeed composite and has xx digits.

- If a cofactor is labelled as Pxx, then it is a prime or probable prime and has $\mathbf{x x}$ digits.

- No odd primitive prime factor of $F_{n}$ or $L_{n}$ was found to divide twice, further strengthening the conjecture that no such prime exists.

Earlier versions of these tables were checked on computers by Michael Morrison and Tim Korb.

As of August 1987 there remain 140 composite Fibonacci cofactors and 10 composite Lucas cofactors in the tables. During 1986 Silverman and Montgomery found numerous factors greater than 20 digits, but none smaller. Based upon numerous runs with ECM, the authors are confident that there are at most 3 undetected factors less than 20 digits.

7. Discussion of Methods. It is still an open question what the best method is to attack a large arbitrary composite number. The authors' experience suggests that the following procedure is perhaps the most reasonable.

As long as the remaining cofactor $N$ is not a probable prime, do the following in order:

(1) Trial division up to some small limit, perhaps $(\ln N)^{2}$.

(2) ECM is generally more effective than $p \pm 1$, but $p \pm 1$ is so much faster that trying it first is worthwhile. A good first set of starting limits is about $10^{4}$ and $10^{5}$. This should perhaps take a couple of minutes on a typical mainframe for (say) an 80-digit number.

(3) ECM should now be tried, using about 5 curves and limits of $10^{4}$ and $5 \cdot 10^{5}$.

(4) If the remaining cofactor is sufficiently small (say up to 60 digits), it should be finished with MP-QS. If the number is larger than this, it is worthwhile devoting more ECM trials with higher limits to it. 
(5) If ECM fails and the number is less than about 70 digits, then MP-QS should now be applied. Seventy digits will take about a day on a typical modern mainframe. One can of course attempt larger numbers with a supercomputer or special hardware. The largest number ever factored with MP-QS, as of December 1986, was an 87-digit cofactor of $5^{128}+1$ using a parallel implementation on a SUN network. That factorization took 3950 total CPU hours, divided among 10 SUN-3's over a period of about 5 weeks.

(6) Finally, if the cofactor is still too large, one can keep trying ECM with higher limits or set the number aside.

TABLE 1

Prime Factors With More Than 25 Digits

\begin{tabular}{|c|c|c|c|c|}
\hline$N$ & Factor & Discoverer & Method & Machine \\
\hline$L_{386}$ & 10245029712795120034405043 & Montgomery & ECM & CDC 7600 \\
\hline$F_{563}$ & 12158771296959377863294133 & gomery & ECM & 7600 \\
\hline$L_{431}$ & 13780495531127210356018421 & & & UVAX/1 \\
\hline$F_{425}$ & 141879543453035643 & & MP-QS & $\mathrm{VAX} / 780$ \\
\hline$F_{507}$ & 212892 & & MP-QS & $/ 8600$ \\
\hline$L_{406}$ & 23670698911880865 & & MP-QS & 780 \\
\hline$L_{371}$ & 3566879698 & & MP-QS & 780 \\
\hline$L_{422}$ & 3630268919283211904258 & & MP-QS & $3 / 75$ \\
\hline$L_{467}$ & 4738105317478219139589 & gomery & ECM & 7600 \\
\hline$L_{320}$ & 62379555831803099867272961 & & CFRAC & Mathilda \\
\hline$F_{837}$ & 1362997727025444376796 & Silverman & MP-QS & $\mathrm{N}-3 / 75$ \\
\hline$F_{445}$ & 1565252892825484 & ilverman & MP-QS & $/ 780$ \\
\hline$L_{471}$ & 47833025812 & & QS & $1 \mathrm{~S}$ \\
\hline$F_{277}$ & 5 & & QS & \\
\hline$F_{517}$ & 6 & erman & MP-QS & $/ 75$ \\
\hline$F_{741}$ & 6696 & & MP-QS & $/ 75$ \\
\hline$F_{597}$ & 1226 & & MP-QS & $3 / 75$ \\
\hline$F_{503}$ & 2430 & & MP-QS & $3 / 75$ \\
\hline$F_{869}$ & 58904 & nery & $\mathrm{ECM}$ & 600 \\
\hline$L_{479}$ & 16372 & & MP-QS & 780 \\
\hline$F_{559}$ & 26093 & & MP-QS & $3 / 75$ \\
\hline$F_{317}$ & 50354 & & QS & 780 \\
\hline$F_{461}$ & 579073 & & 25 & $-3 / 75$ \\
\hline$F_{633}$ & 1923474742854608312 & & MP-QS & $-3 / 75$ \\
\hline$L_{326}$ & 57300568099 & $\mathrm{Sil}$ & MP-QS & $-3 / 75$ \\
\hline$F_{971}$ & 61980260725951458 & mery & $p-(5 / p)$ & CDC 7600 \\
\hline$L_{412}$ & 10904143353831684635 & omery & ECM & CDC 7600 \\
\hline$L_{344}$ & 1403981099723321029379913948641 & Silverman & MP-QS & VAX/780 \\
\hline$L_{482}$ & 5373430329122468821883671012169 & Montgomery & ECM & CDC 7600 \\
\hline$L_{377}$ & 9220407243723719942154317888399 & Silver & MP-QS & SUN-3/75 \\
\hline$F_{489}$ & 55010483350408487052485570744297 & Silver & MP-QS & SUN-3/75 \\
\hline$F_{663}$ & 542202788462733966380018208818089 & & MP-QS & SUN-3/75 \\
\hline$F_{681}$ & 1316534463290847218590097513564 & & MP-QS & SUN-3/75 \\
\hline$L_{430}$ & 1517416544639719175645264380247 & & MP-QS & SUN-3/75 \\
\hline$F_{383}$ & 15318508443810774614619603643 & & MP-QS & SUN-3/75 \\
\hline$F_{427}$ & 249495868964998482 & Silve & MP-QS & SUN-3/75 \\
\hline & 227693725298545340302283668318476481 & Montgomery & ECM & CDC 7600 \\
\hline
\end{tabular}


The present practical limit of technology seems to be about 16 digits for prime factors found by Pollard Rho, 18 digits for Brent's variation of Pollard Rho, and 25 digits for ECM. The $p \pm 1$ methods occasionally have huge successes where a factor over 25 digits is found; for example, these methods could have found the 29-digit factor of $L_{479}$ with a little more effort. However, factors of 18 to 20 digits are more typical. The CFRAC method has been demonstrated for products up to $10^{64}$, QS for products up to $10^{71}$, and MP-QS for products up to $10^{87}$. This comparison is not quite fair, however, because the CFRAC and QS results were achieved either on a supercomputer or on special purpose hardware, while the MP-QS results were achieved on a network of SUN's [17], [18].

Table 1 lists all of the known nonlargest primitive prime factors of $F_{n}$ or $L_{n}$ having more than 25 digits. The cofactor of each of these, when it is composite, is assumed to have at least one prime factor exceeding the factor listed. Each entry includes the discoverer, the method of discovery, and the machine used. In the "machine" column the notation "UVAX/1" is an abbreviation for Micro-VAX/1.

Acknowledgments. The authors wish to thank their institutions for providing the extensive computer time required for this work. Silverman extends special thanks to Tom Caron for writing the networking software that made the parallel implementation of MP-QS possible. Montgomery extends special thanks to Mike Mitchell and Richard Milton for the many hours they spent running his CDC 7600 jobs. All of us extend special thanks to Andrew Odlyzko for providing many of our primality tests and to Dave Cantor, Tim Korb, D.H. and Emma Lehmer, Michael Morrison, J. L. Selfridge, Peter Weinberger, Hugh C. Williams, and Marvin C. Wunderlich for their assistance over the years in helping to create these tables.

Department of Mathematics

University of Arizona

Tucson, Arizona 85721

UNISYS

2525 Colorado Avenue

Santa Monica, California 90406

MITRE Corporation

Burlington Road

Bedford, Massachusetts 01730

1. J. BRILlHART, "Fibonacci century mark reached," Fibonacci Quart., v. 1, 1963, p. 45.

2. J. BRILlHART, "Some miscellaneous factorizations," Math. Comp., v. 17, 1963, pp. 447-450.

3. J. BRILlhaRT, D. H. LehmeR \& J. L. SElFRIDGE, "New primality criteria and factorizations of $2^{m} \pm 1$," Math. Comp., v. 29, 1975, pp. 620-647.

4. J. BRILlHART, "UMT of $F_{n}$ for $n \leq 3500$ and $L_{n}$ for $n \leq 1750$," Math. Comp., submitted to unpublished tables.

5. J. Brillhart, D. H. Lehmer, J. L. Selfridge, B. Tuckerman \& S. S. Wagstaff, JR., Factorizations of $b^{n} \pm 1, b=2,3,5,6,7,10,11,12$ Up to High Powers, Contemp. Math., vol. 22, Amer. Math. Soc., Providence, R.I., 1983.

6. H. Cohen \& H. W. Lenstra, JR., "Primality testing and Jacobi sums," Math. Comp., v. 42, 1984, pp. 297-330.

7. J. A. DAvis \& D. B. Holdridge, Most Wanted Factorizations Using the Quadratic Sieve, Sandia Report SAND84-1658, 1984. 
8. J. L. GerVer, "Factoring large numbers with a quadratic sieve," Math. Comp., v. 41, 1983, pp. 287-294.

9. D. JARDEN, Recurring Sequences, 3rd ed., Riveon Lematematika, Jerusalem, 1973.

10. D. H. LEHMER, "An announcement concerning the Delay Line Sieve DLS 127," Math. Comp., v. 20,1966 , pp. $645-646$.

11. H. W. LenStRA, JR., "Factoring integers with elliptic curves," Ann. of Math. (To appear.)

12. Peter L. Montgomery, "Modular multiplication without trial division," Math. Comp., v. 44, 1985, pp. 519-521.

13. Peter L. Montgomery, "Speeding the Pollard and elliptic curve methods of factorization," Math. Comp., v. 48, 1987, pp. 243-264.

14. M. A. MORRISON \& J. BRILlHART, "A method of factoring and the factorization of $F_{7}$," Math. Comp., v. 29, 1975, pp. 183-205.

15. T. NAUR, Integer Factorization, DAIMI PB-144, Computer Science Department, Aarhus University, Denmark, 1982.

16. T. NAUR, "New integer factorizations," Math. Comp., v. 41, 1983, pp. 687-695.

17. Robert D. Silverman, "The multiple polynomial quadratic sieve," Math. Comp., v. 48, 1987, pp. 329-339.

18. ROBERT D. Silverman, "Parallel implementation of the quadratic sieve," The Journal of Supercomputing, v. 1, 1987, no. 3.

19. H. C. Williams, "A p+ 1 method of factoring," Math. Comp., v. 39, 1982, pp. 225-234.

20. H. C. Williams \& J. S. JUDD, "Some algorithms for prime testing using generalized Lehmer functions," Math. Comp., v. 30, 1976, pp. 867-886.

21. H. C. WILliaMS, private communication. 


\title{
Supplement to Tables of Fibonacci and Lucas Factorizations
}

\author{
By John Brillhart, Peter L. Montgomery and Robert D. Silverman
}

\section{TABLE 2 FIBONACCI FACTORIZATIONS $2<n<1000, n$ odd}

\section{$\underline{\text { Prime Factors }}$}

32

55

713

9 (3) 17

1189

13233

$15(3,5) 61$

171597

1937.113

$21(3,7) 421$

2328657

25 (5) $5^{*} .3001$

$27(3,9) 53.109$

29514229

31557.2417

$33(3,11) 19801$

$35(5,7) 141961$

37 73.149.2221

$39(3,13) 135721$

412789.59369

43433494437

$45(3,5,9,15) 109441$

472971215073

49 (7) 97.6168709

$51(3,17) 6376021$

53 953.55945741

$55(5,11) 661.474541$

$57(3,19) 797.54833$

59353.2710260697

614513.555003497

$63(3,7,9,21) 35239681$

$65(5,13) 14736206161$

67269.116849 .1429913

$69(3,23) 137.829 .18077$

716673.46165371073

$73 \mathbf{9 3 7 5 8 2 9 . 8 6 0 2 0 7 1 7}$

$75(3,5,15,25) 230686501$

$77(7,11) 988681.4832521$ 

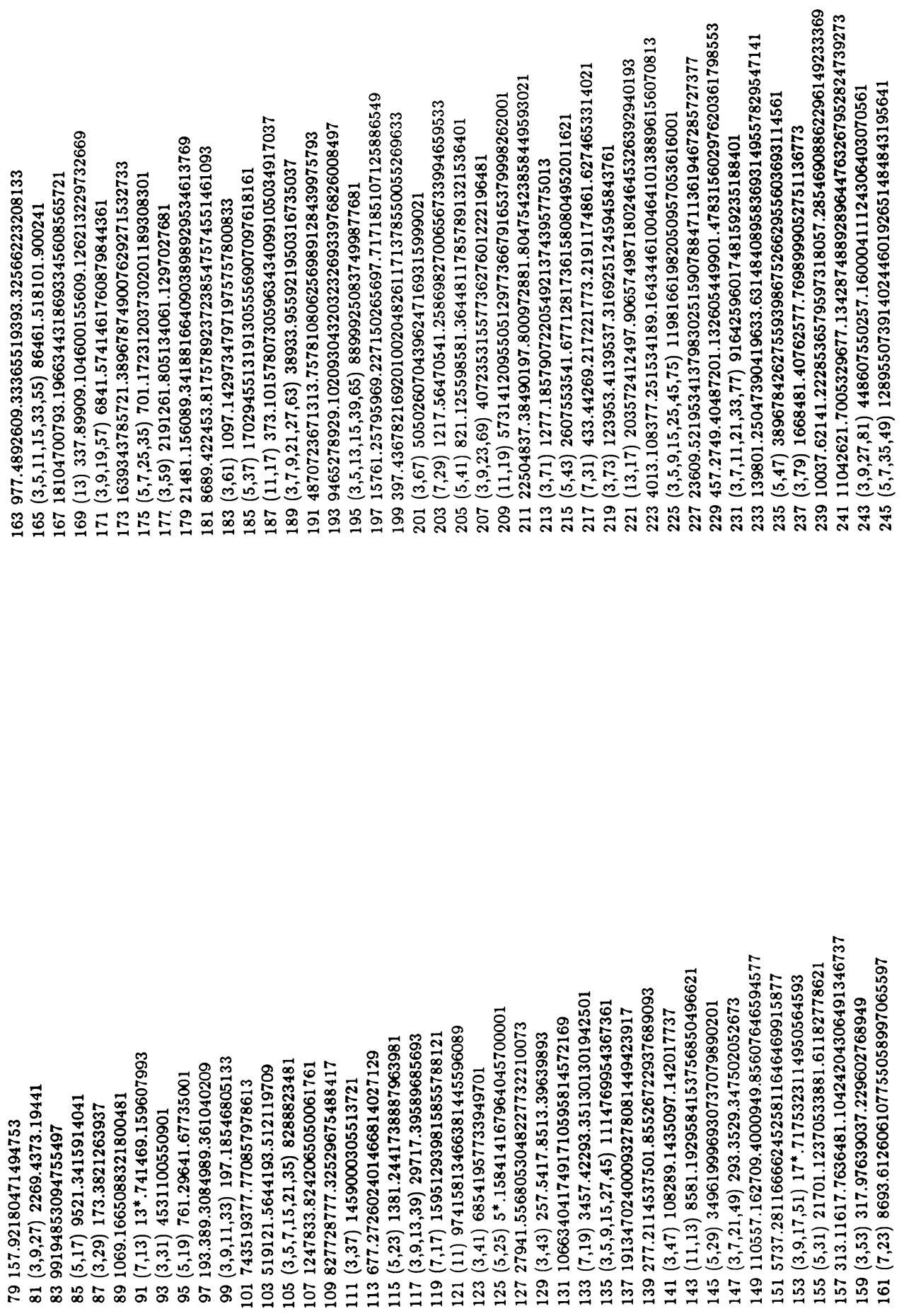

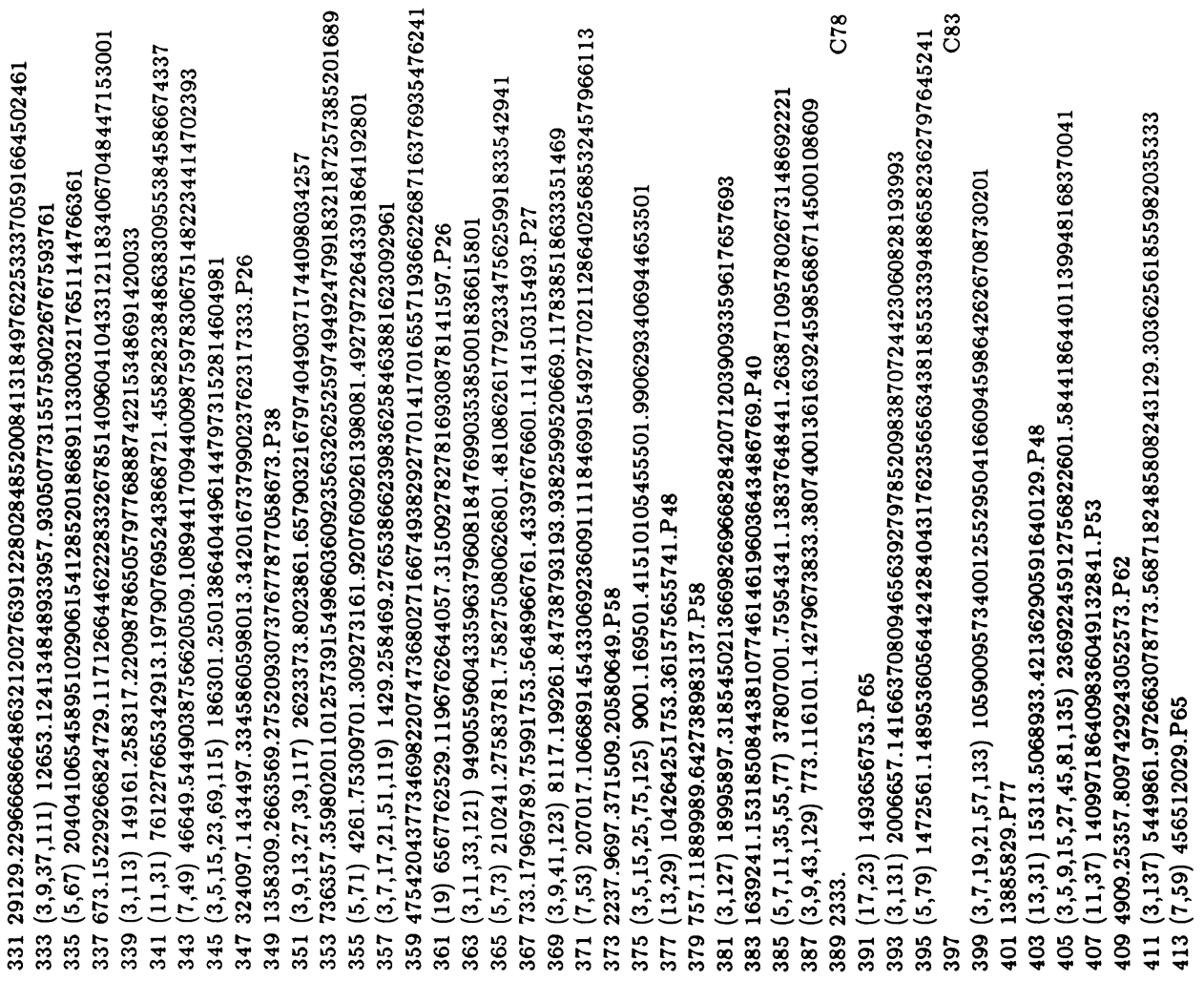

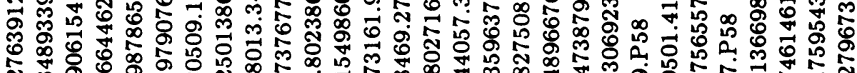

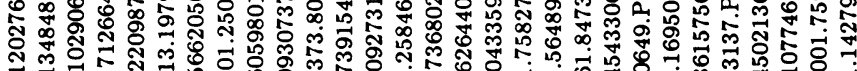

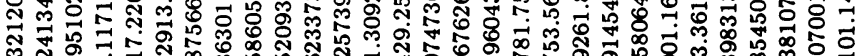

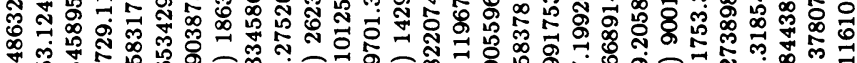

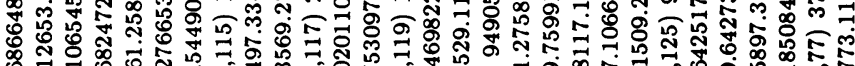
o ज न

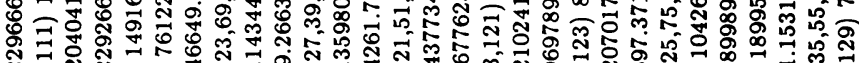

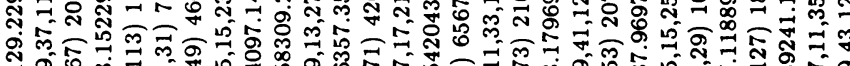

क०

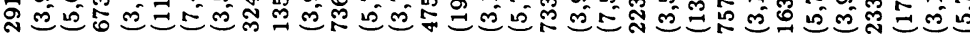

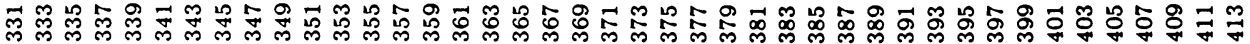

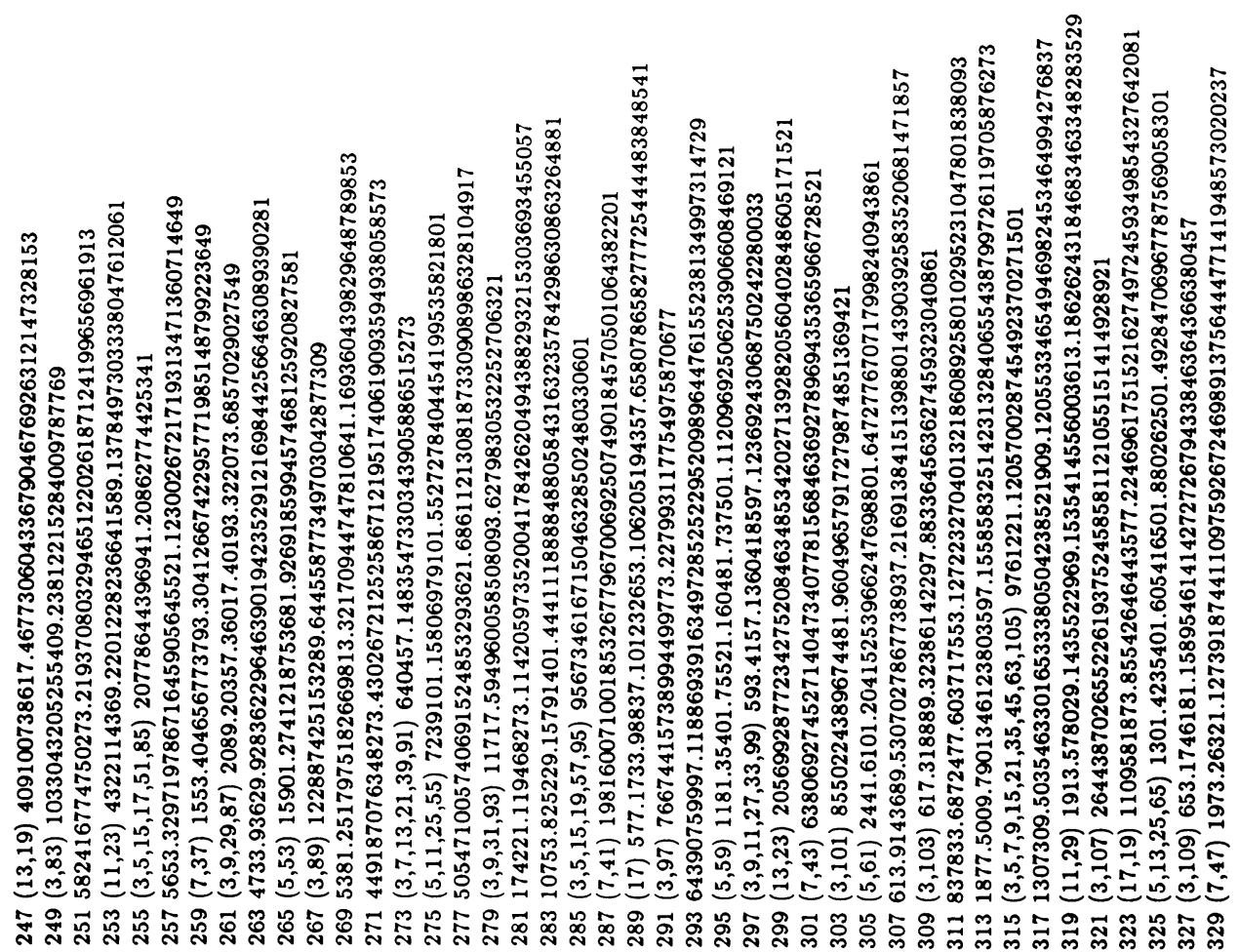



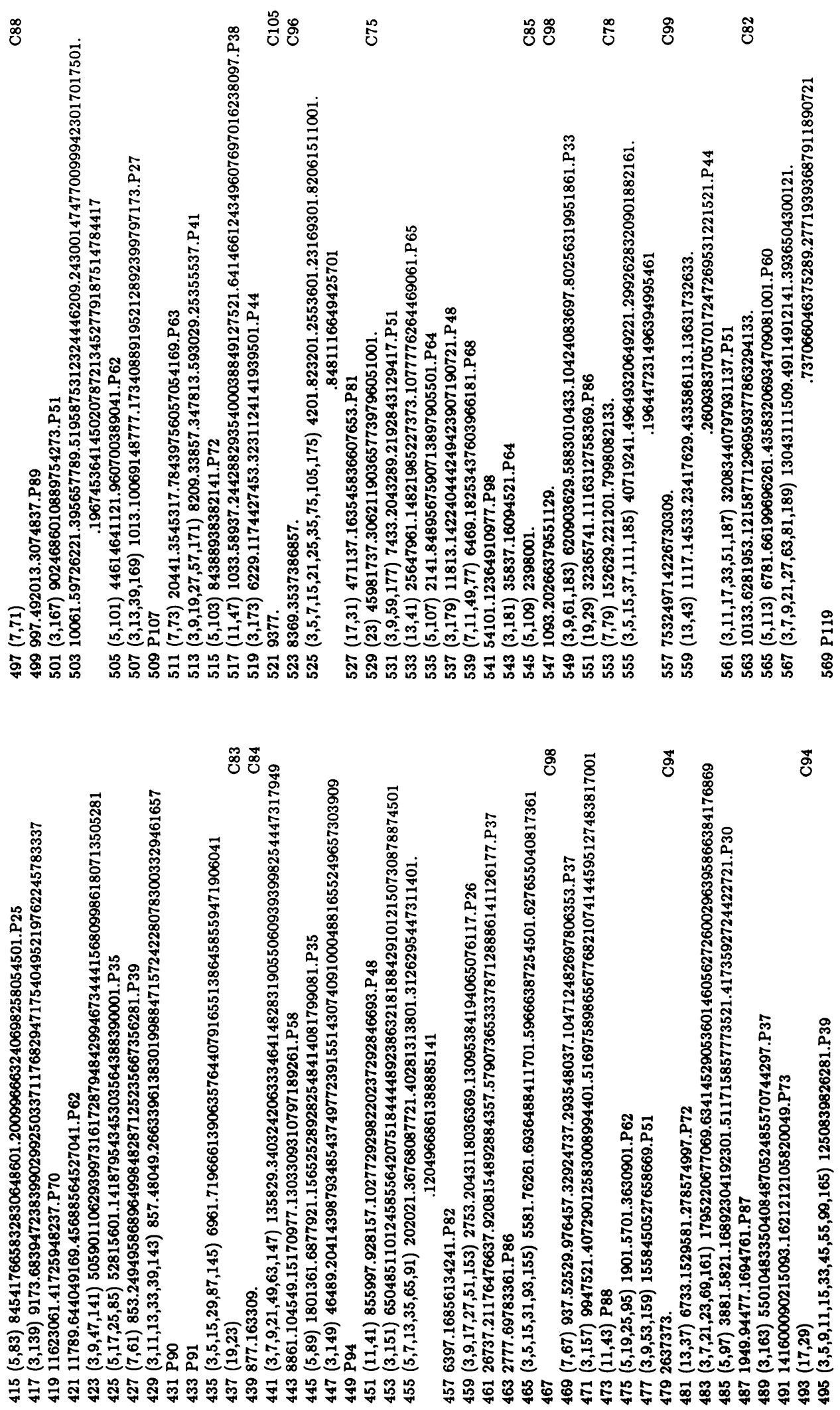

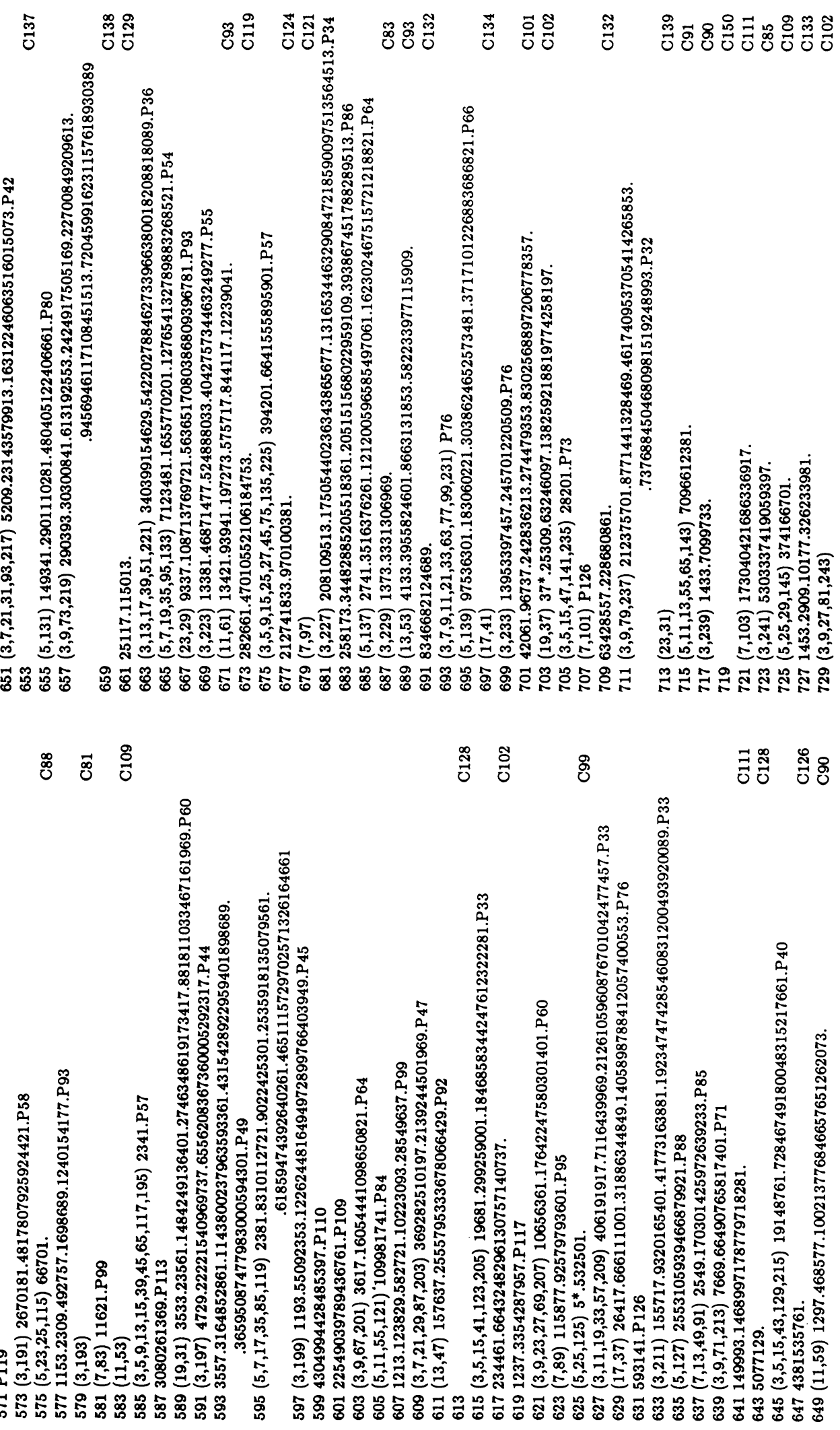


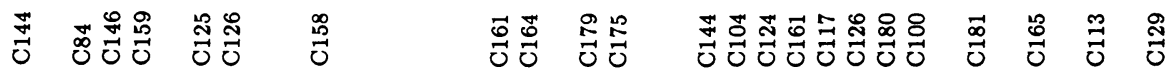

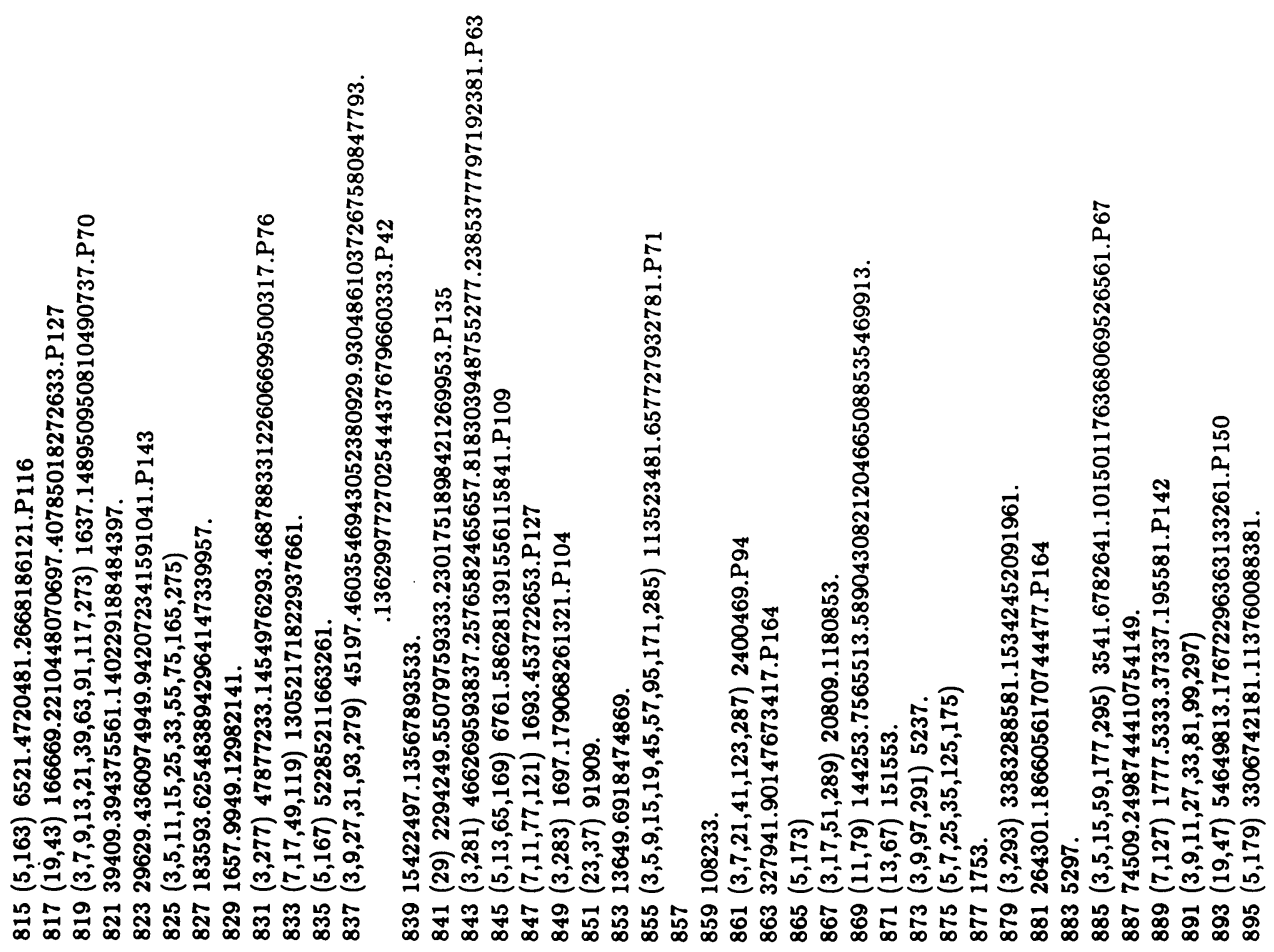

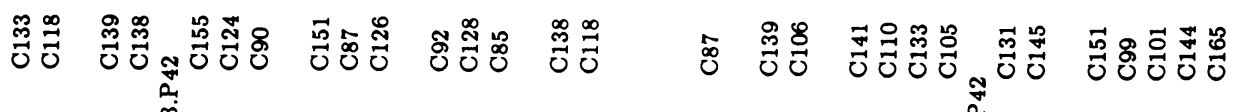

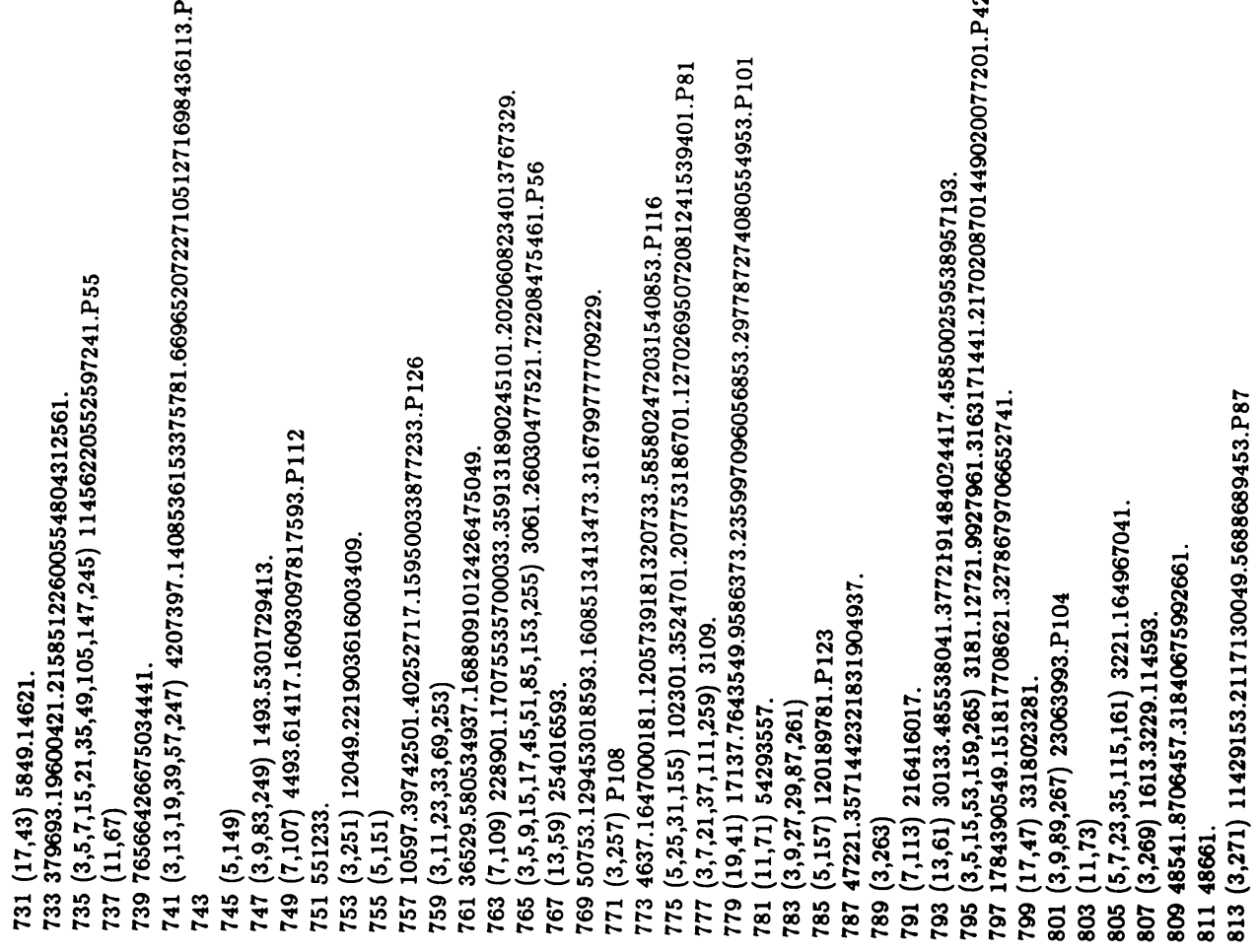




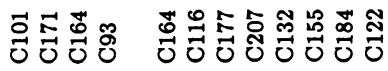

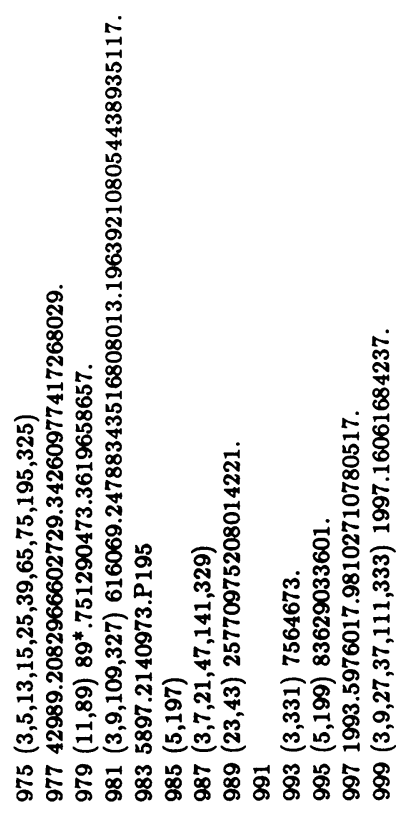

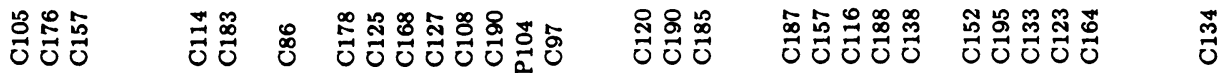
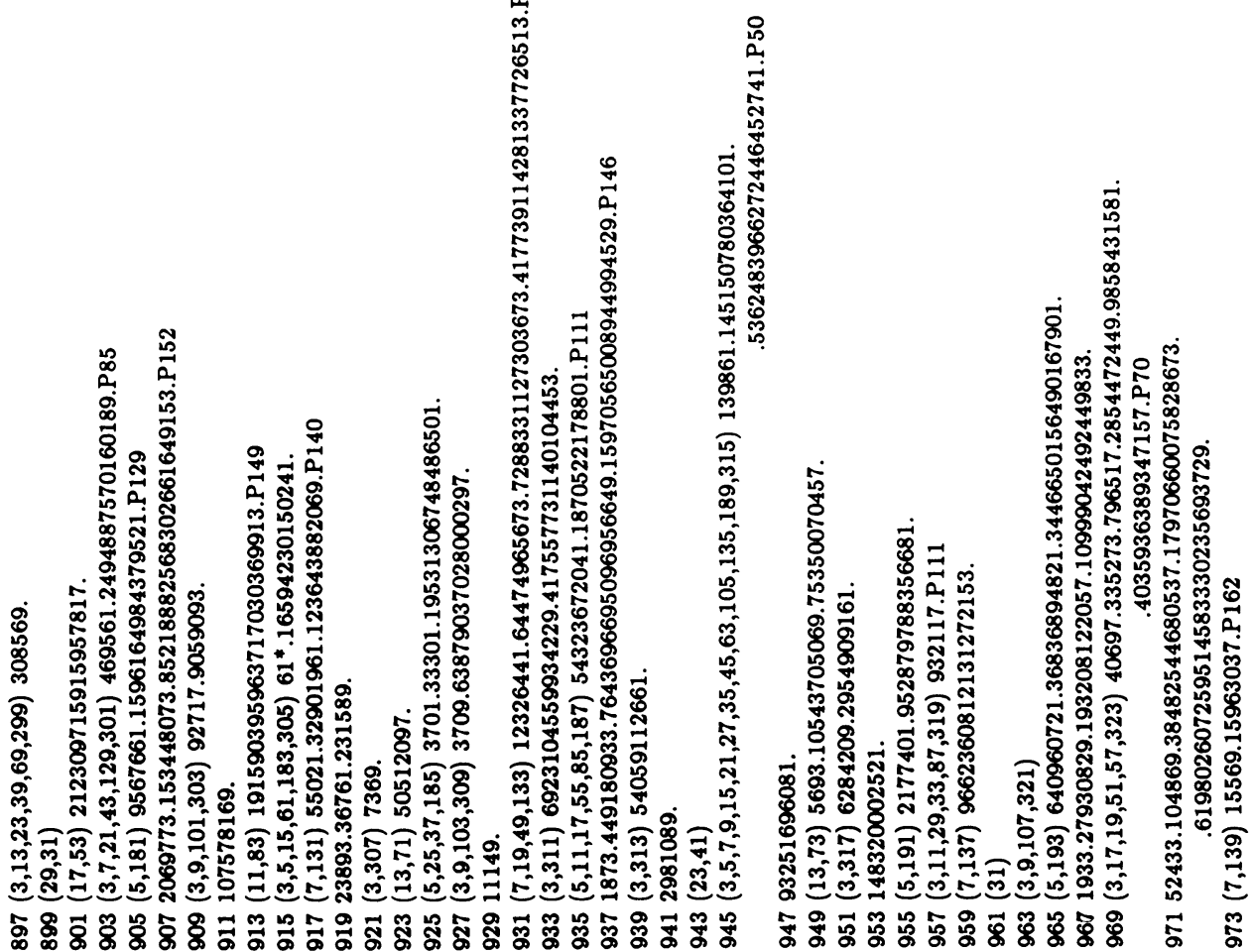

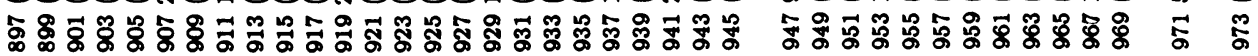




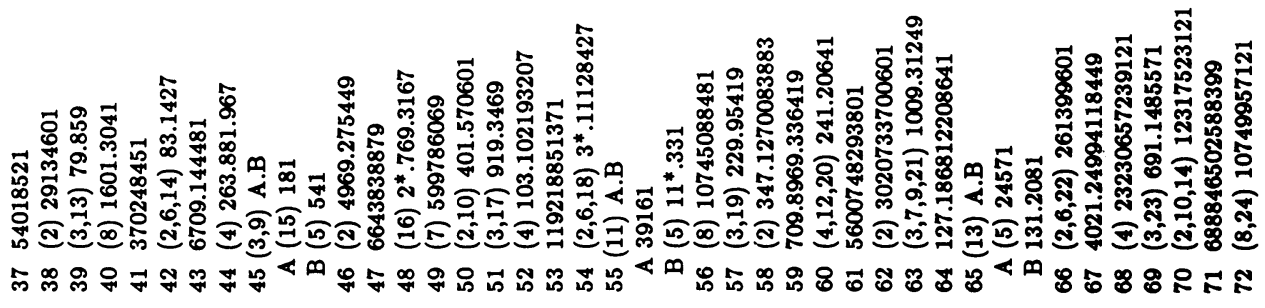
순 

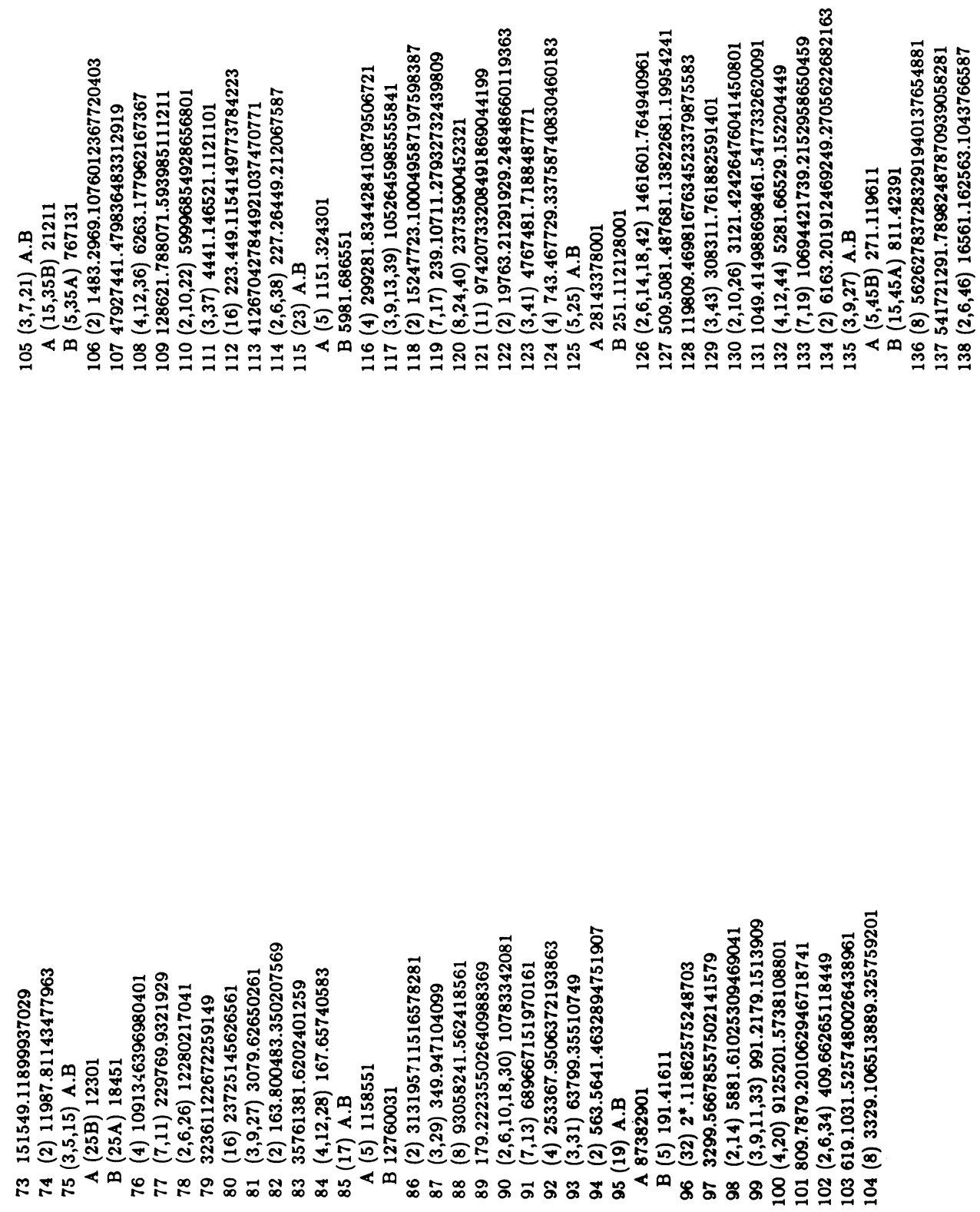

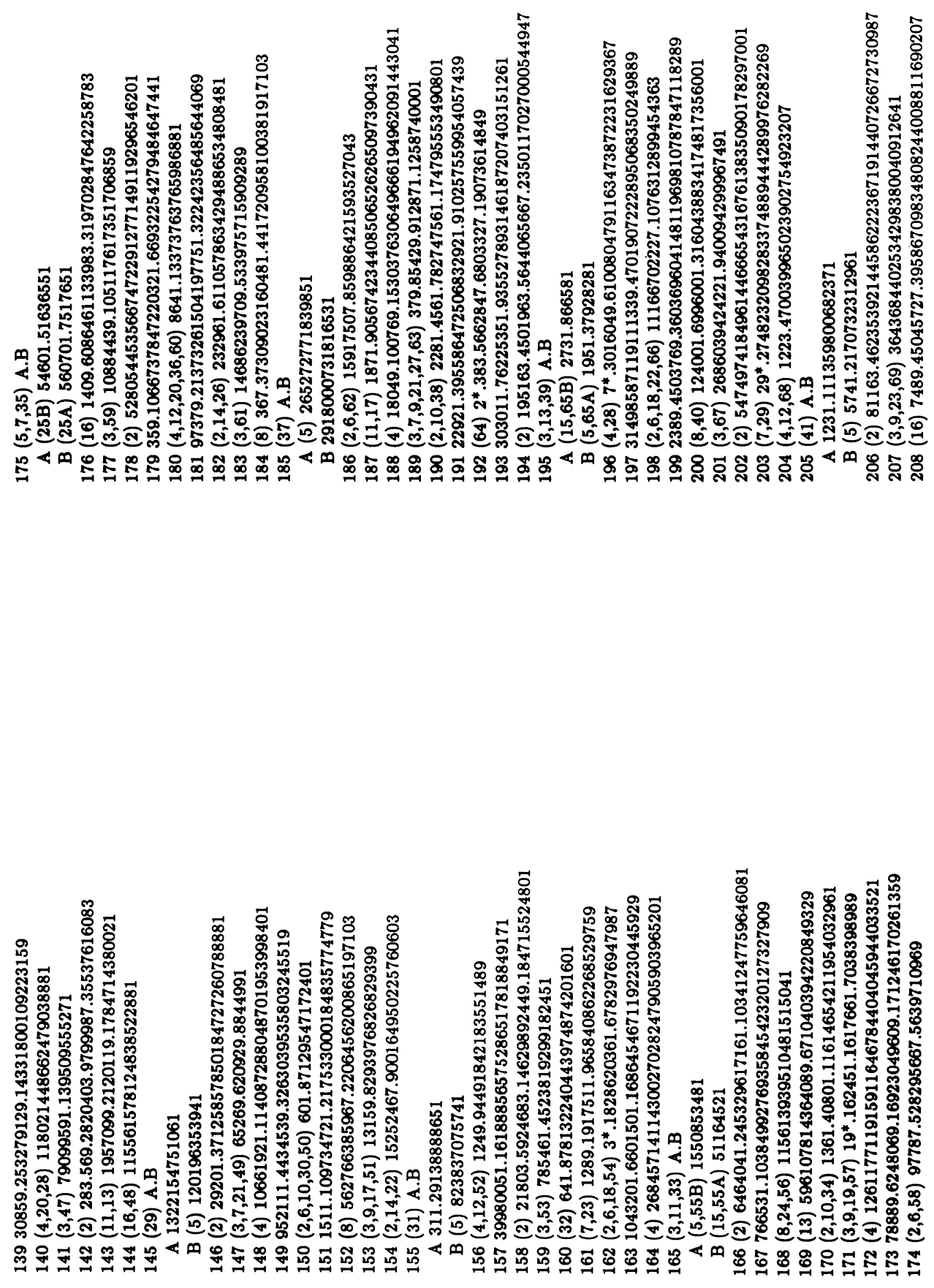


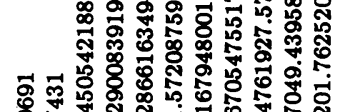

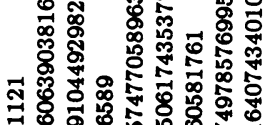

1.

密

象

数震

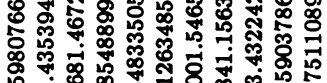

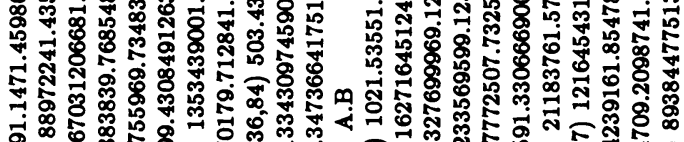

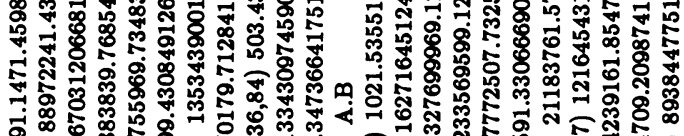

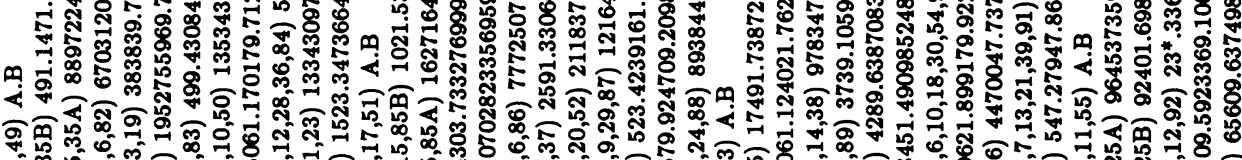

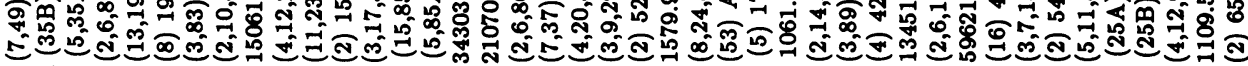

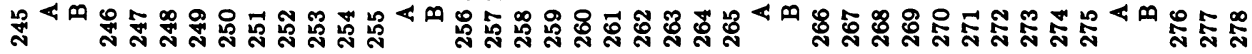

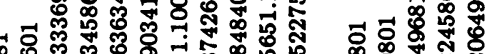

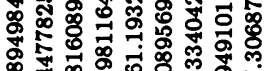

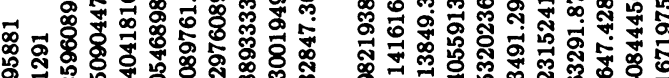

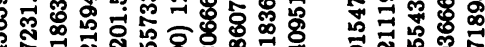
중 성

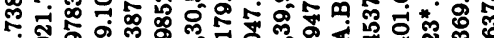
(n)

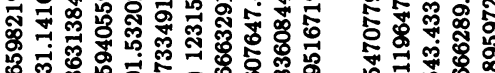



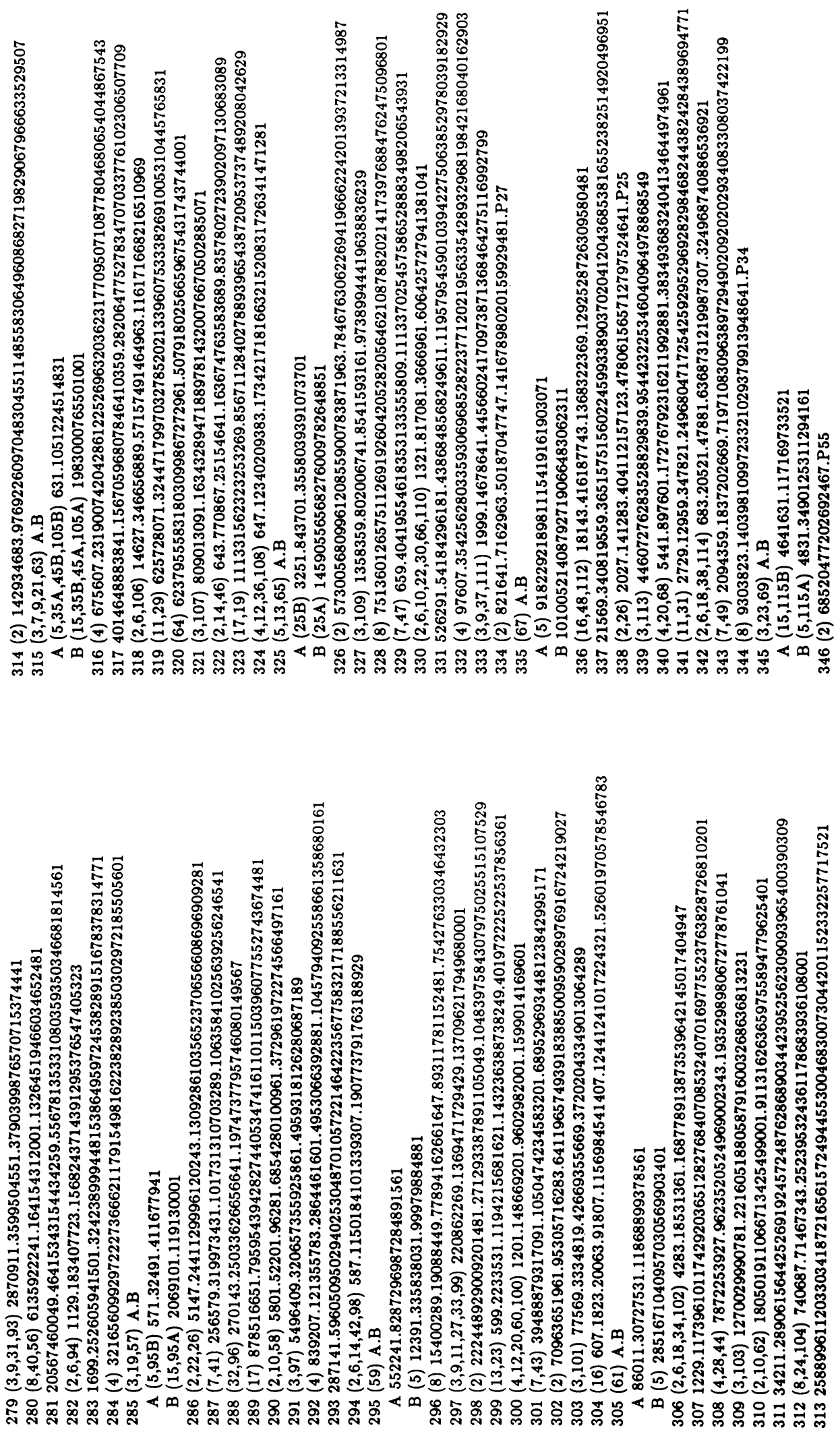
$\mathscr{\infty}_{0}^{\infty}$
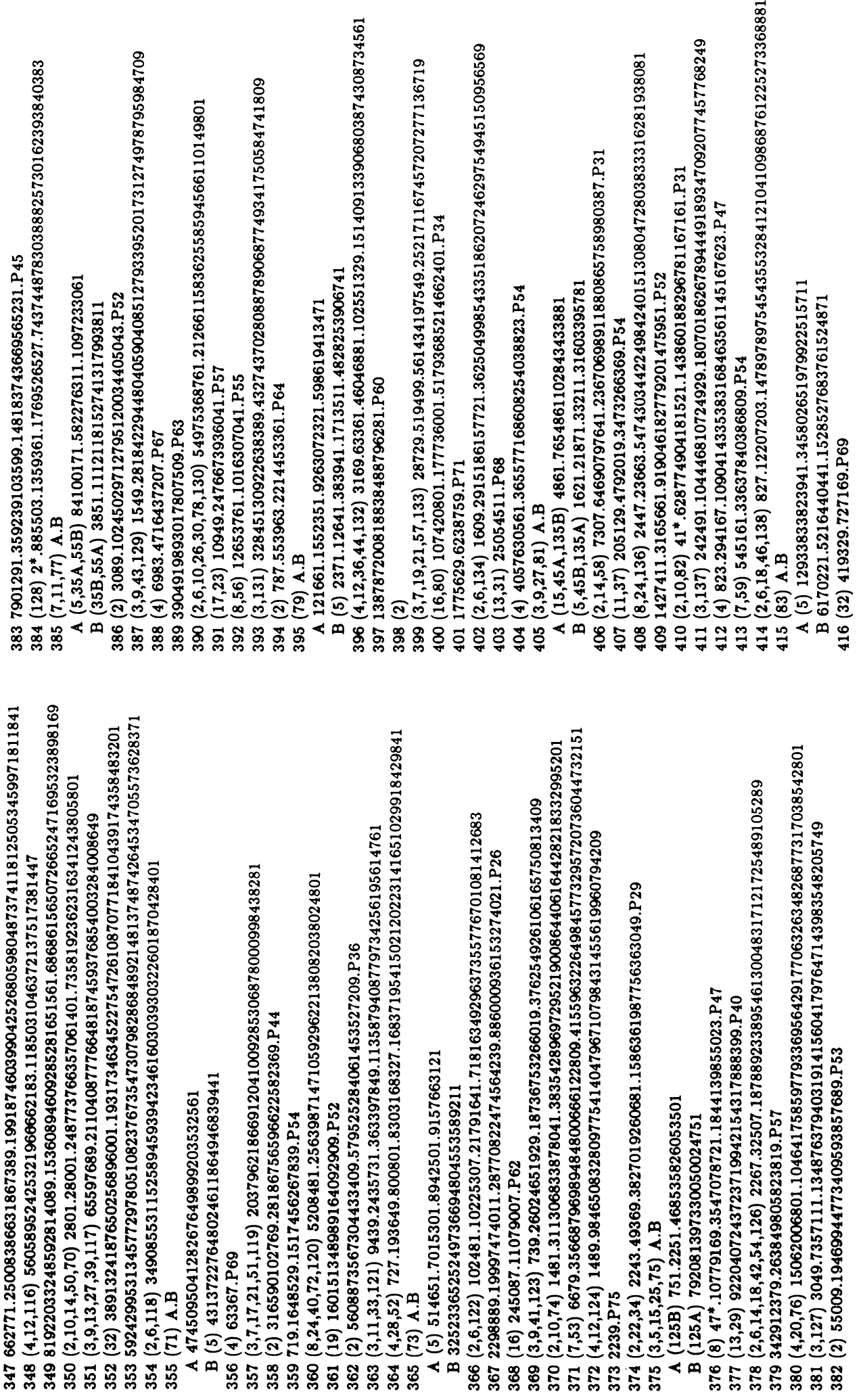

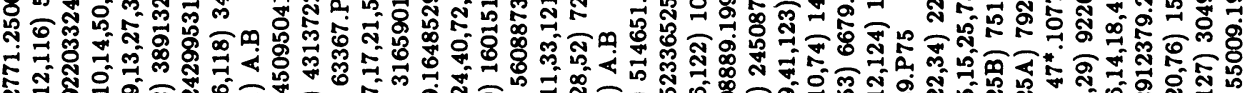

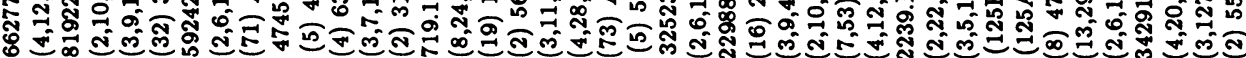

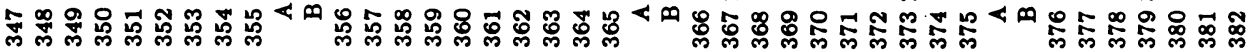



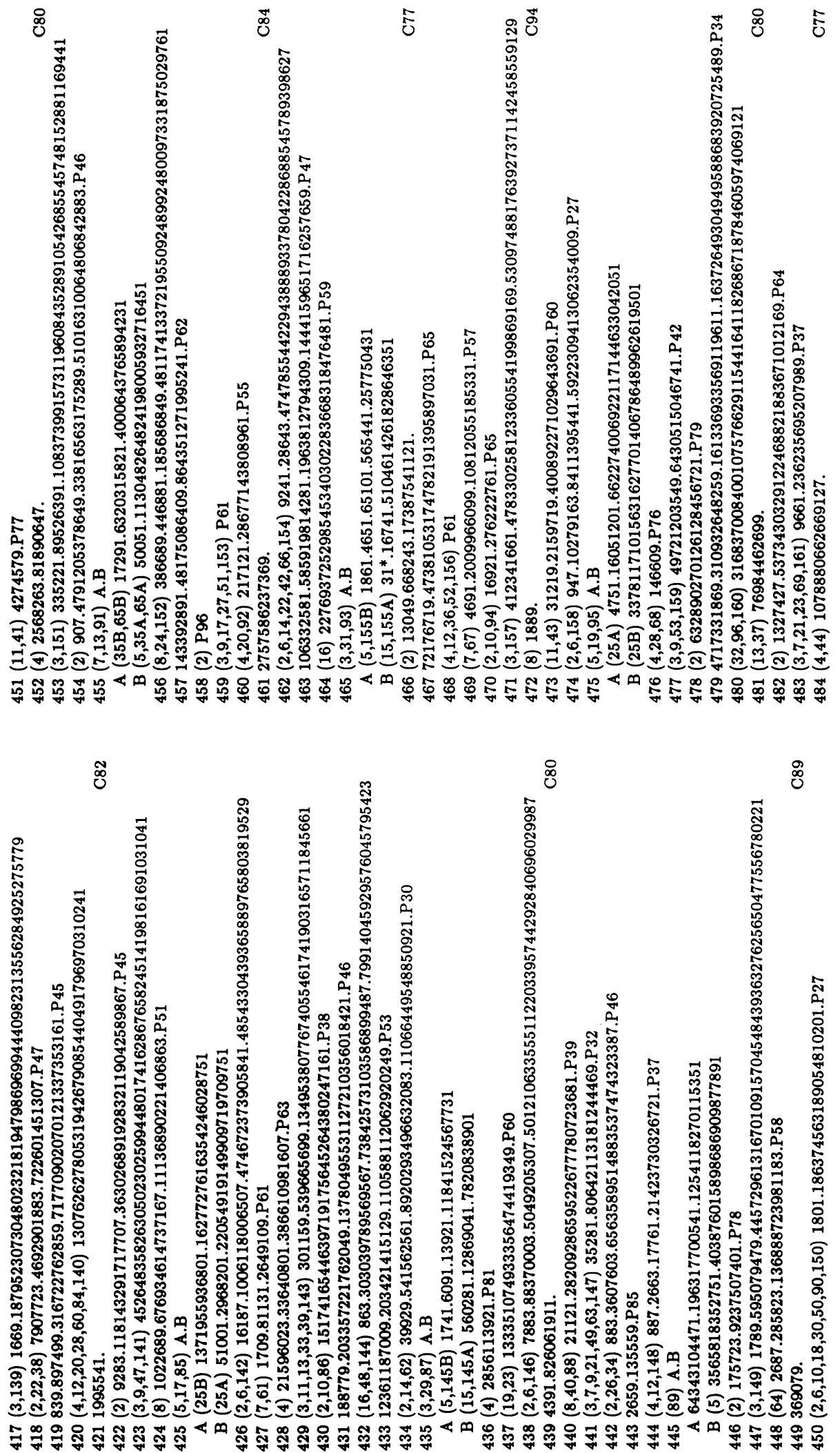


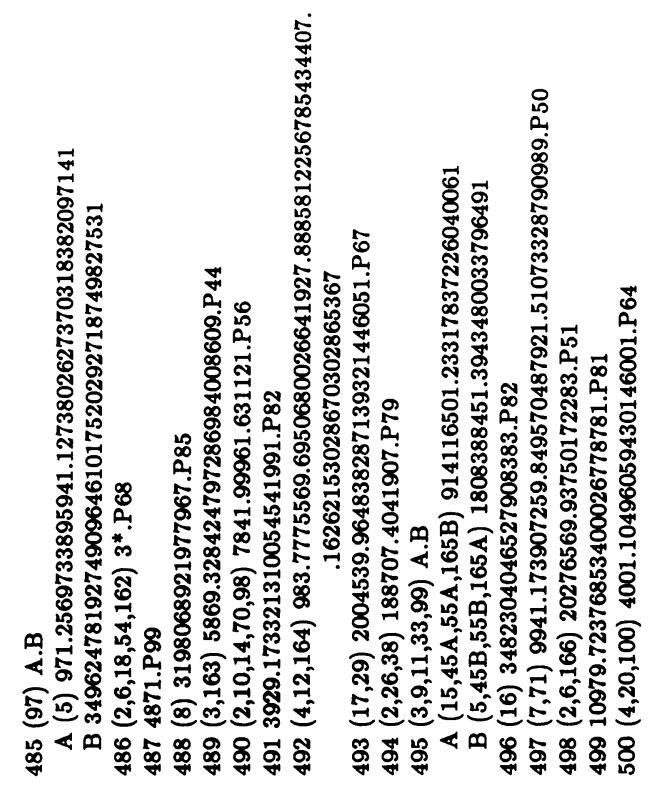

